\title{
Impact of COVID-19 on Transgender Women and Hijra: Insights from Gujarat, India
}

\author{
Apurvakumar Pandya ${ }^{1}$. Alex Redcay ${ }^{2}$
}

Accepted: 21 June 2021 / Published online: 19 July 2021

(c) The Author(s), under exclusive licence to Springer Nature Switzerland AG 2021

\begin{abstract}
Protecting vulnerable populations from the COVID-19 pandemic is a strategic imperative, especially when considering the potential vulnerability it has on a wide range of marginalized communities. Transgender women and hijra individuals who struggle to cope financially, mentally, or physically are vulnerable groups that require attention during the COVD-19 pandemic. Using a qualitative descriptive research design, 12 transgender women and hijra persons participated in semistructured telephone interviews during India's lockdown phases (1.0 and 2.0) of the COVID-19 pandemic. A snowball sampling technique was used to recruit study participants. Authors identified five themes: knowledge and practice; psychological domain; health conditions; individual and community experiences; and support. Findings offer broad insights into bolstering health concerns and access to healthcare services from public health and human rights perspective. An access to adequate healthcare services is a fundamental human right. The COVID-19 pandemic response without a human rights framework will have a major impact on the progress of the Sustainable Development Goals. Future studies of this population will illuminate unique considerations for healthcare workers while providing care to this neglected group.
\end{abstract}

Keywords Transgender women $\cdot$ Hijra $\cdot$ LGBTQ $\cdot$ COVID-19 pandemic $\cdot$ Gujarat $\cdot$ India

\section{Introduction}

Transgender women and hijra ${ }^{1}$ persons remain marginalized. They are often deprived of healthcare entitlements because of their gender identity (Ming et al., 2016). The fear of being stigmatized and ridiculed by healthcare professionals discourages them from using available healthcare services (Chakrapani, 2016; Ganju \& Saggurti, 2017), which during the pandemic, is leaving them at an increased risk of not being tested or treated for COVID-19 (Deb, 2020). COVID19 has posed physical as well as mental health risks to this marginalized population as well. Deb (2020) rightly drew attention to the distresses faced by transgender individuals in India during the COVID-19 pandemic. The concern is not just about the lack of healthcare facilities but also the lack

Apurvakumar Pandya

apandya@iiphg.org; drapurvakumar@gmail.com

1 Indian Institute of Public Health, Gandhinagar, Gujarat, India

2 Millersville University, Millersville, PA, USA of dialogue about the needs and inclusion of transgender individuals in the healthcare system.

The government of India declared a 69-day lockdown from March 23, 2020, until May 31, 2020. Table 1 lists the lockdown phases that were enforced to help contain the spread of COVID-19 in India.

Lockdown was gradually lifted from June 2020 onward. During the lockdown phases 1.0 and 3.0, public places were closed, and police restricted people's movements. In addition, physical distance and proper hygiene practices regarding handwashing and coughing were highly recommended to prevent further spread. By April 30, 2020 (lockdown phase 2.0), India had 33,050 cases and 1074 dead. Ahmedabad had 4395 cases, with 214 dead, giving a higher fatality rate (4.9\%) than the rest of the country (3.2\%). This lockdown period was strict, and the entire public health system was engaged in addressing the COVID-19 pandemic with little focus on other essential healthcare services. After the lockdown was gradually lifted, the cases of COVID-19

\footnotetext{
${ }^{1}$ Hijra is a term commonly used in India to refer to a third gender person, not entirely male or female. Some would consider hijra and transgender as equivalent in definition. For the purpose of this paper, participants have self-identified as either hijra or transgender women and we have honoured the individual's choice in terminology.
} 
Table 1 Lockdown enforcements by the government

\begin{tabular}{ll}
\hline Lockdown phases & Period \\
\hline Lockdown phase 1.0 & March 24 to April 14, 2020 \\
Lockdown phase 2.0 & April 15 to May 3, 2020 \\
Lockdown phase 3.0 & May 4 to May 17, 2020 \\
Lockdown phase 4.0 & May 18 to May 31, 2020 \\
\hline
\end{tabular}

Source: Ministry of Health and Family Welfare

increased. On September 13, 2020, the Ministry of Health and Family Welfare reported 970,116 confirmed cases of COVID-19 and 92,290 dead, resulting in around 9.5\% fatality rate in the country (Ministry of Health \& Family Welfare, 2020).

Numerous studies have highlighted specific health problems that affect the transgender population and barriers that limit their access to healthcare services. For example, the prevalence of human immunodeficiency virus (HIV) in transgender women and hijra persons is the second highest (3.14\%) across all most-at-risk population groups (i.e., people injecting drugs, female sex workers, and men who have sex with men) and remain higher among all adults in India (0.22\%) (National AIDS Control Organization, 2017; National AIDS Control Organization \& ICMR-National Institute of Medical Statistics, 2020). Additionally, the incidence of physical violence, substance use, and various adverse mental health outcomes have been reported in this special group in India (Chakrapani et al., 2017, 2019; Hebbar \& Singh, 2017; Humsafar Trust, 2012; Jayadeva, 2017; Thompson et al., 2019).

Transgender-friendly healthcare services in India are nearly non-existent. Healthcare workers continue to treat gender incongruence with the sex assigned at birth as a psychological deviation or consider it a mental illness (Chakrapani et al., 2016). Barriers to access healthcare services have been well-documented. Saraswathi and Praveen (2015) estimated that $20 \%$ of the transgender individuals in India have unmet transgender-specific healthcare needs.

Many State governments, including Gujarat, have constituted a Transgender Welfare Boards (TWB) following the Supreme Court of India in 2014 recognized transgender persons as a third gender and held that the rights of transgender persons should be safeguarded and that they should be able to access schemes and entitlements. However, TWBs have not yet yielded concrete actions in addressing the social protection needs of transgender people, including health care, housing, education, and employment in the State. Moreover, TWB's work in the time of pandemic remains unknown. Transgender individuals continue to struggle with: stigma and discrimination at healthcare facilities; a lack of gender-sensitive treatment protocols; low health literacy among transgender women and hijra persons; low literacy; gender-based violence; poor healthcare-seeking behaviors; socioeconomic barriers; lack of health insurance; and exclusion from social protection schemes (Pandya \& Redcay, 2021).

Even before the COVID-19 pandemic, researchers indicated an intolerance toward those who do not conform to heteronormative identities, transphobia, and negative attitudes toward transgender women and hijra persons stigmatize and marginalize these identities (Thomson et al., 2019). Therefore, it is expected that the COVID-19 pandemic may exacerbate more negative attitudes toward transgender individuals, and these internalized negative attitudes may prevent transgender individuals from accessing public healthcare services.However, their current situation during the COVID-19 pandemic has not yet been reported. Since the beginning of the pandemic, various studies (Armitage \& Nellums, 2020; Banerjee et al., 2020; Hall et al., 2020; Roy et al., 2020; Spoorthy et al., 2020) have been conducted to understand the impact of the pandemic on people who are infected, affected people, and other vulnerable people, especially healthcare workers. However, studies on the effect of the COVID-19 pandemic on transgender women and hijra persons are scarce. Therefore, it is essential to understand how transphobia and normative social standards may have played a role in accessing healthcare by transgender individuals in India during the COVID-19 pandemic. The present research studied participants' knowledge about COVID-19, risk perception, socioeconomic and health conditions, and their experiences of access to healthcare services during the lockdown phase (1.0 and 2.0) of the COVID-19 pandemic Gujarat.

\section{Method}

We used a qualitative descriptive methodology to develop detailed descriptions of transgender women and hijra persons' experiences during lockdown (Kim et al., 2017). Based on the tenets of naturalistic inquiry, the qualitative descriptive design is a flexible approach that is particularly useful for getting a straightforward description of a phenomenon and answering questions relevant to practitioners (Kim et al., 2017). We have carried out telephonic interviews using a semi-structured interview guide with openended questions allowing for follow-up. Authors developed the interview guide to gather data based on their experience working with this special group and the targeted literature review. The data analysis yielded a description of the data rather than an in-depth conceptual description or development of the theory (Miles \& Huberman, 1994). 


\section{Participants and Recruitment}

Participants were recruited through snowball sampling using inclusion and exclusion criteria. Participants could participate if they were (a) a transgender woman or hijra individual living in Ahmedabad (the largest city in Gujarat State), (b) 18 years old or older, (c) part of gharana (housing tradition where hijra individuals shelter in groups or clusters and each gharana follows their rules and rituals) or part of guru-chela (mentor-disciple) tradition independent of gharana, and (d) willing to provide consent. Individuals selfidentified as kothis (effeminate same-sex sexually-oriented men) who cross-dress, transgender women outside of the gharana or guru-chela tradition, and those who declined to provide consent were not included in the study.

As transgender women and hijra individuals in India constitute a stigmatized and closed community, those who had previously sought psychological support were contacted and were requested to refer other transgender women or hijra individuals for the study. Further, a local community-based organization (CBO) working with transgender women and hijra individuals in Ahmedabad city was also asked to refer participants to the study. Referred participants were screened by the researchers for inclusion and completed the consent process. After participants' consent, telephonic interviews were conducted. The in-depth interviews were approximately 40 to 85 min long, and participants were also asked to refer their peers to the study.

\section{Research Instrument}

A small yet growing body of literature has documented the potential of in-depth telephone interviews as a viable option for qualitative research (Cachia \& Millward, 2011; Lechuga, 2012) and collecting narrative data on sensitive topics such as sexuality and traumatic life experiences specific to the lesbian, gay, bisexual, transgender, and queer (LGBTQ) community (Drabble et al., 2016; Law, 2015). In addition, telephone interviews appear to increase a perceived sense of anonymity and are well-suited to gather data on sensitive topics (Irvine et al., 2013; Trier-Bieniek, 2012).

The interview guide included 30 questions which were organized into five themes. (1) The Knowledge and Practice domain included questions about the participants' knowledge about COVID-19, their perceived susceptibility to COVID-19 infection, and their use of preventive measures. (2) The Psychological domain included questions investigating participants' psychological reactions to the lockdown and how they were coping with it. (3) The Individual and Community Experiences domain explored community reactions, food security, and livelihood. (4) The Health domain comprised questions about participants' mental, physical, and sexual health concerns and healthcare-seeking behaviour. (5) The Support domain explored participants' family, gharana, community organizations, and partners' supportiveness. These five themes guided the semistructured interview guide. The English-based interview schedule was translated into Gujarati (a language spoken in Ahmedabad, Gujarat). The Gujarati interview guide was also back-translated to ensure reliability.

\section{Data Collection}

Semi-structured telephone interviews were conducted with transgender women and hijra residing in Gujarat between March 25 to April 30, 2020. Of the total study participants contacted (15), two transgender women and one hijra declined participation in the study interview for personal reasons. Hence, a total of 12 participants were interviewed. All interviews were conducted in Gujarati, a colloquial language for the participants. The text of the interviews was translated and transcribed into English. The researchers ensured that no culture-specific information was lost in the translation process. Some words and concepts were transcribed in Gujarati when the researchers felt that translation might harm the cultural relevance or meaning of the participants' answers.

\section{Ethical Considerations}

All procedures performed in the study were aligned with ethical standards. The study was approved by the Ethical Review Committee of the local community-based organization. Consent of all study participants was sought. Additionally, pseudonyms have been given to participants, and locations were de-identified to preserve anonymity.

\section{Data Analysis}

A narrative thematic analysis (Braun \& Clarke, 2006; Cortazzi, 2014) of the interviews consisted of generating, revising, and categorizing codes in relevant themes. Based on an initial round of coding, text segments were identified and further evaluated by researchers for frequency and the theoretical centrality of their salience. One researcher was involved in the coding process while the other researcher reviewed the coding. Both researchers had categorized all codes and grouped them into one theme. At every stage of coding, a researcher kept memos to account for any emerging theoretical topics or issues later resolved by discussion and mutual agreement. Participants' answers are presented verbatim in italics. Furthermore, selected quotes representing each theme are presented. 
Table 2 Sociodemographic characteristics of the study's participants

\begin{tabular}{ll}
\hline Sample characteristics & $f(\%)$ \\
\hline Gender identity & \\
Transgender women & $8(67.6)$ \\
Hijra & $4(33.3)$ \\
Region & \\
Urban (city) & $7(58.3)$ \\
Semi-urban (periphery of the city) & $5(41.6)$ \\
Religion & \\
Hindu & $9(75)$ \\
Muslim & $3(25)$ \\
Education & \\
Primary (up to 8th grade) & $9(75)$ \\
Secondary (10th grade) & $2(16.6)$ \\
University education (under-graduation) & $1(8.3)$ \\
Average income (monthly) & 13,600 INR $(\$ 185)$ \\
Income range (monthly) & $4500-26,000$ \\
& INR $(\$ 61.24-$ \\
& $\$ 353.85)$ \\
\hline
\end{tabular}

\section{Results}

\section{Sociodemographic Characteristics}

Nearly two thirds of the study participants $(n=8)$ identified as transgender women, while one third $(n=4)$ were self-identified hijra persons. ${ }^{2}$ The average age of the study participants was $33.6 \pm 10.8$ (range 19-53 years). Details of the education and livelihood of the study's participants are provided in Table 2.

Hijra persons tend to live together with their Guru in one home compared to transgender women who primarily live either with a partner or live alone. Out of the four hijra persons, three lived with their $G u r u,{ }^{3}$ whereas one lived alone. In the case of the transgender women participants, three stayed alone in rented rooms in the slum; two lived with their Guru; two lived with their respective male partners.

\footnotetext{
${ }^{2}$ Hijra individuals in India would be considered transgender women in the USA. Many countries use equivalent terminology.

${ }^{3}$ Hijra community in India largely has a hierarchical structure which is different from general society. Hijra individuals often live together in a household that is headed by "guru" (leader and mentor). Several chela (disciples) live under one "guru." Hijra community follows norms of respective the "gharana" (tradition) they belong to.
}

\section{Knowledge and Practice}

\section{Knowledge About COVID-19}

Most participants $(n=11)$ were aware of how COVID-19 spreads. However, many of them $(n=10)$ were unaware of precautions for preventing COVID-19 and treatment centers. The following excerpt reflects the same,

"COVID-19 is deadly disease, spread with touching, coughing of COVID-19 infected persons...I am unaware of Government's COVID-19 treatment services nearby my residence." (Anjali, 26 years old, transgender woman).

"I think sore throat, fatigue, and diarrhea are symptoms of Coronavirus infection...it [coronavirus] is dangerous, people die if contracted coronavirus." (Asha, 53 years old, hijra person).

The major sources of information about COVID-19 among study participants were from television news and social media. Most participants $(n=7)$ were dependent on WhatsApp messenger, whereas others $(n=4)$ were updating themselves about COVID-19 from TV news. In addition, one transgender woman reported having access to the Ahmedabad Corporation Website and State Government Web site for the latest updates.

"I get information about Coronavirus from WhatsApp [social messenger]." (Varsha, 19 years old, transgender woman).

"...television is there at my home, [which] provides information about coronavirus." (Asha, 53 years old, hijra person).

\section{Perceived Susceptibility of COVID-19 Infection}

Few participants, both transgender women $(n=2)$, believed they were at risk of contracting COVID-19 because they had interacted with many people as part of their routine work just before lockdown. However, most participants $(n=10)$ perceived considerably low risk of contracting the coronavirus. The following statements reflect the kinds of religious beliefs that affect how they perceive the virus' risk.

"...this virus is here to wipe away evils in this world... people with good karma need not worry. And tell me, I do not go out, how can I get infected..." (Pallavi, 22 years old, transgender woman).

"We are a disciple of Behchara Mataji [Goddess Behchara $\mathrm{Ji}$ in Gujarat]. If we are true Bhakt [devotee], she will save us." (Asha, 53 years old, hijra person).

\section{Use of Preventive Measures}

A belief that "coronavirus cannot infect us" was prevalent among transgender women and hijra participants. Five of 
them believed that COVID-19 could not infect them as they worship "Bahuchara Mataji" [goddess Bahuchara], and she will protect them. The following excerpts give context for why they do not follow safety precautions. As one hijra participant described,

"We live together...almost 6-7 in one room. Many amongst us have a cough and cold but are not aware of correct [coughing] practices...I know, but being a $\mathrm{Chela}^{3}$ [disciple], no one listens to me... our Guru [master or teacher] believes that nothing will happen to us as we are a devotee of Bahuchara Mataji” (Tamanna, 24 years old, hijra person).

\section{Psychological Domain}

\section{Psychological Reactions to Lockdown}

Most participants $(n=11)$ expressed anxiety and fear due to the lockdown. Anxieties were fuelled by losing livelihood options $(n=6)$, difficulties in staying home all the time $(n=2)$, their HIV infection $(n=2)$, and tuberculosis disease $(n=1)$. The following excerpt describes anxiety around disclosure of HIV status and access to medication.

"I was diagnosed with HIV last year, and my partner does not know about it. My medicines will stock out in the next ten days. I am worried about how I will go to the antiretroviral treatment (ART) centre and what if my partner will come to know about it." (Monika, 33 years old, transgender woman).

In terms of economic needs, hijra persons $(n=4)$ managed daily needs from their savings. Gurus were taking care of the needs of their Chelas as most of them were living in the vicinity of their Gurus except one. However, the economic conditions of transgender women were worse. Transgender women had limited savings, and their livelihoods depended on begging in trains and highway toll plazas, dancing during weddings or baby showers, marriage, and sex work. The following excerpt echoes the same.

"We have no income.... running cashless; all savings are used-up...food stock is about to complete. We are in extreme need of money or food assistance...I am worried about the future." (Anjali, 26 years old, transgender woman).

Most transgender women and hijra persons live in congested and densely populated areas. One hijra person had excessive fear of COVID-19 infection as she saw her community and many other places were designated as COVID19 hotspots-localities with a high number of COVID-19 cases by the local administration. She was worried about herself, her Guru, and her peers.

“...many people from our locality have been infected with COVID-19. I fear of contracting COVID-19. I am worried about my Guru, my partner, and my friends." (Pooja, 38 years old, transgender woman).

\section{Coping}

When asked about coping with the current situation, most participants did not know how to deal with the present situation. Common coping was praying to Mataji (Goddess), increased alcohol use, reduced medicine doses, and borrowed money. For example, a transgender woman on medication for HIV reduced her dosage (from two pills a day to one pill a day) to continue medication until the lockdown is lifted.

"I am on HIV medication. My medicines are about to complete ... I have started taking one pill a day [instead of two] to prolong the treatment till I visit ART centre for a check-up and get medicines." (Monika, 33 years old, transgender woman).

Three transgender women reported borrowing money from private moneylenders who charged higher interest rates (3\% per month) to meet their needs. Few transgender women $(n=2)$ made alcohol at home, whereas others, who were substance-dependent, reportedly went outside and broke lockdown rules searching for their desired substance.

Hiding their symptoms of fever and cough was common. A participant said,

"Few persons had cough and fever, but when health personnel came for checking, they were not allowed to enter the home and informed all are healthy...this is due to fear of contracting with COVID-19 and fear of being stigmatized." (Anjali, 26 years old, transgender woman).

Due to a lack of awareness and suspended anti-retroviral treatment (ART) services, transgender women and hijras living with HIV were anxious and feared contracting COVID19. For example, one participant living with HIV stated that, "I fear contracting COVID-19 as my ART medicines are about to complete... my immunity will [gradually] weaken and [thus] I will contract COVID-19."

\section{Mental Health Concerns}

About three participants expressed mental health concerns. Financial distress, the uncertainty of the COVID-19 pandemic, intimate partner violence, and a lack of access to healthcare services were key factors that adversely impacted their mental health. A transgender woman said,

"I feel like dying rather than losing a relationship. My partner, who drives an autorickshaw, persistently beats me and gets aggressive. The financial crisis has worsened our relationship as we both cannot earn. I am tired of life, and COVID-19 has put me into trouble." (Pooja, 38 years old, transgender woman).

A hijra person undergoing gender affirmative surgery also had a tough time. She experienced an anxiety-related delay in her ongoing treatment and worried about its consequences. 
"Medicines for hormone replacement therapy is completed and not able to meet a doctor for further treatment. I am worried about possible ill effects of interrupted treatment." (Priya, 28 years old, transgender woman).

See the earlier section for descriptions of incidents of fear and anxiety.

\section{Health Conditions}

\section{Physical and Sexual Health Concerns}

Many participants $(n=5)$ reported suffering from chronic diseases such as diabetes $(n=1)$, asthma $(n=1)$, tuberculosis $(n=1)$, and HIV $(n=2)$. One transgender woman participant was undergoing a gender-transitioning procedure. Six participants - three hijra persons and three transgender women - were alcohol-dependent, and three who were consuming tobacco had difficulty managing their cravings for the substance. A hijra person mentioned, “...everything is closed down. People cannot get 'tamaku' [tobacco leaves for chewing] or "deshi daaru" [indigenous alcohol or country liquor]. I find it difficult to manage cravings ...I get sweating and headache." (Hira, 43 years old, hijra person).

\section{Decreased Access to Healthcare}

Access to healthcare was a significant challenge for participants. About six participants needed healthcare services but could not access them. This resulted in fear and anxiety among many of the study's participants, as evident from the following excerpts.

"I am suffering from diabetes, used to take medicines daily. Since last two days medicines stoked out. I am worried for my health. How can I go out to get medicines?" (Aradhana, 48 years old, transgender woman).

"I was currently undergoing Hormone Replacement Therapy. I had an appointment next week, but this lockdown has stopped my treatment." (Priya, 28 years old, transgender woman).

In an earlier section, we saw fear among participants for not accessing ART services. In addition, fear of discrimination at healthcare facilities also precluded them from seeking COVID-19-related testing or treatment services.

\section{Internalized Anti-trans Discrimination in Public Healthcare Facilities}

Transgender women and hijra persons reported experiencing discrimination at healthcare facilities even before the COVID-19 pandemic. Most participants $(n=8)$ preferred private healthcare providers over public healthcare services due to prevalent negative attitudes toward their gender identity. One transgender woman described anticipated discriminatory attitudes in public healthcare facilities. She said, "healthcare providers treat us differently...do not talk with us properly, and do not even examine properly." Another transgender woman stated, “...not only the infected transgender person will have to deal with the physical and emotional trauma of being infected by the virus, but also will face negative attitude of the healthcare providers as well as fellow patients." (Aradhana, 48 years old, transgender woman).

A hijra participant said, "we often face negative attitudes from doctors and fellow patients..." Another hijra person described their experience of discrimination at a hospital:

"Recently, my community member was admitted in the male ward despite being in women's clothing and had 'nirvan' [removal of male external genitalia]. Naturally, such an incident traumatizes an already infected with Corona." (Manju, 41 years old, hijra person).

These quotes reflect the participants' internalized stigma and discrimination which discourages them from accessing healthcare services.

\section{Individual and Community Experiences}

\section{Livelihood and Food Insecurity}

Lockdown left many transgender community members unable to earn an income. It has been substantiated by one of the transgender persons interviewed. The participant said,

"We used to earn about 300-400 every day by begging and during auspicious functions. But all these have stopped now. We are worried about meeting our needs" (Asha, 53 years old, hijra person).

With little savings and social security benefits, many face the massive challenge of depleting food supplies. The Indian State Government had started providing food support (wheat, rice, pulses, and sugar) through their public distribution system (Economic Times, 2020). Surprisingly, none of the study participants received food support. One participant reported, "none of us have received food from the Government." Many of them were not aware of such food provision, which is reflected in one of the participant's words, "we are not aware of such a scheme." Two participants mentioned not having government identity cards such as Aadhar Card and Below Poverty Line Card to access the benefits of such programs. A transgender woman said,

"Most of the people in my community lack government identity proofs such as Aadhaar Card, Driving License, Passport, Permanent Account Number, Ration card (either below poverty line card or above poverty line card), Voter identity cards and therefore, we are excluded from various social 
security schemes of the Central or State government." (Anjali, 26 years old, transgender woman).

\section{Support}

Most transgender women were denounced at home by their families and were either living alone, living with their Guru, or living with their partner. They reported tough times due to a lack of support system, unlike what the hijra community has experienced. On the other hand, hijra persons reported support from the Guru.

"We are at Hijra Gharana is like a family. We stay together in all difficulties of life. I am grateful to my Guru who takes care of all Chelas, and we take care of her just like a mother." (Hira, 43 years, hijra person).

Lockdown had curtailed the support for activities by community-based organizations working with transgender women and the hijra population in the city. Nevertheless, many participants $(n=5)$ expected CBO to reach out to them and provide food or monetary assistance in such critical times. Furthermore, support from their partners was negligible. Few participants helped their partners in terms of financial support, and they feel they cannot expect support from their partners as they will not be able to help them financially.

"In this tough time, what partner can do for us? In fact, we do for our partner. I gave half of my savings to partner to take care of his family. Now I am penniless." (Mohini, 29 years old, transgender person).

\section{Discussion}

The study highlights factors that increase the vulnerability of transgender women and hijra in the times of COVID- 19 . Lack of adequate knowledge, perceptions of low-risk, nonadherence to COVID-19 safety measures, and fear of declaring COVID-19 symptoms among the study's transgender women and hijra participants were noted. These altogether may collectively affect their access to healthcare services during the pandemic. Although with limited sample size to draw any firm conclusions, reasons for fear, low risk-perception, and non-adherence to COVID-19 safety measures and fear can be attributed to religious beliefs, internalized anti-trans discrimination, rumours, and lack of access to reliable information. Future research in this area with a larger sample size can provide more insights. Future research in this area with a larger sample size can throw more insights.

\section{Vulnerability of Transgender Women and Hijra Persons to COVID-19}

It is important to note that strategies most recommended to control the spread of COVID-19 include physical distancing, proper hygiene regarding coughing, frequent handwashing, and wearing masks (World Health Organization, 2020; Ministry of Health \& Family Welfare, 2020), are not easy for transgender individuals to follow who live in highly dense communities with insecure housing and poor sanitation. Often many transgender individuals have chronic illnesses such as diabetes, asthma, HIV, and tuberculosis (Chakrapani et al., 2017; Muraleedharan et al., 2018; NACO, 2017; Ortiz, 2016; Stop TB Partnership, 2018). The use of tobacco and alcohol was worrisome. More than half the sample in the present study consumed tobacco and alcohol. This makes them more vulnerable to mental health problems as well as affects their respiratory system (CDC, 2020). Furthermore, they are unlikely to access the government's support as many do not have government identity cards, a bank account, or health insurance. Hence, they remain vulnerable to violations of their rights to access healthcare services. For all the reasons mentioned above, transgender women and hijra persons are likely to be significantly impacted by the COVID-19 pandemic more than their cisgender peers. Thus, they should be considered vulnerable, echoing the recommendation of the Lancet Global Health editorial (Editorial, 2020), and interventions should be deemed urgent.

\section{Barriers to Essential Healthcare Services}

The study has highlighted barriers to healthcare access, including mental health services. As illustrated by study participants describing their experiences testing positive for COVID-19 and being admitted to male health wards, transgender persons who are COVID-19 positive might encounter twofold stigma: firstly because of their gender identity and COVID-19 positive status, and secondly because of their past experiences with the healthcare system might drive transgender women and hijra persons away from reporting COVID-19 symptoms, further spreading the virus (James et al., 2016; Ortiz, 2016). The history of stigma and discrimination of transgender women and hijra in the healthcare system can internalize stigma and discrimination. It further prohibits them from reporting their symptoms of the coronavirus infection as well as accessing public healthcare facilities. These factors, if unaddressed, may result in a high COVID-19 fatality rate in transgender women and hijra persons. Thus, responses to COVID-19 should make efforts to ensure access to COVID-19 testing and care services. The need for separate LGBT COVID-19 isolation wards have been reported (Praveen, 2020) and argued that separate wards would resolve the long-standing problem of discrimination (Deb, 2020).

Inaccessibility to general healthcare, including sexual health services during the lockdown, might have adversely affected the lives of transgender women and hijra persons. The situation may be worse in rural areas where health 
services are just limited to health centres and hospitals (Singh, 2020). Hence, transgender women and hijra persons' health needs must be integrated into any pandemic preparedness plan. Moreover, healthcare service providers should show sensitivity toward transgender women and hijra persons and their healthcare needs. At the same time, the need for raising health literacy, awareness of individual rights, available healthcare facilities, and government health schemes such as health insurance cannot be undermined.

\section{Socioeconomic Conditions and Food Insecurity}

Since most transgender individuals solely depend on dealing with others in society, the pandemic has adversely impacted their livelihood during the lockdown. In order to fulfil their daily needs during the lockdown, they borrow from multiple loaning sources, usually from private lenders who charge higher interests and thus remain in debt.

As noted, most transgender women and hijra persons do not possess government documents such as an Aadhaar Card and a Below Poverty Line Card (Elavati, 2016; Sharma, 2018). The absence of these documents essentially excludes them from receiving social protection benefits, especially food security and financial aid from the government during the lockdown and beyond. These needs are also should be considered for pandemic preparedness plans and execution.

\section{Lessons to Learn}

There are many lessons to learn from these findings. The government must make gender-disaggregated COVID-19 data (including transgender women, transgender men, and hijra persons) available in the public domain. At the same time, policymakers must ensure equitable and meaningful representation of transgender individuals in designing interventions and policymaking. It is imperative that Transgender Welfare Board take cognizance of the issue and aid social protection schemes, including healthcare services, housing, and employment related opportunities to the transgender population.

The government can capitalize on this opportunity to address this population's basic needs such as food security, livelihood, and health issues during public health emergencies such as COVID-19. Providing gender-affirmative psychological services, continuing supply of hormonal therapy, and other gender-affirming procedures during a pandemic is vital.

More studies and data are needed to illuminate the unique considerations for provisioning, encouraging access, and delivering healthcare services. Evidence generated from studies can inform policymakers and administrators to take appropriate actions to bridge policy gaps and improve the implementation of social protection schemes and healthcare services. Hegde (2020) rightly proposes that academic and government institutions should prioritize research on the impact of COVID-19 on transgender individuals, and investment in gender-conscious and gender-responsive research is needed (Hegde, 2020).

In summary, the authors note that while the entire world is combatting the COVID-19 outbreak, transgender individuals struggle with their means of survival, social elimination, access to healthcare services, and layers of discrimination enforced on them because of their unique gender identity. Access to adequate healthcare services is a fundamental human right. The COVID-19 pandemic response without a human rights framework will have a major impact on the 2030 Sustainable Development Goals (Avafia et al., 2020). Essentially, we argue that access to essential healthcare services, food security, and psychosocial support for transgender individuals is also crucial in the fight against COVID-19.

\section{Limitations of the Study}

The urgency of the situation incurs some limitations to the study. The study focused on transgender women and hijra persons only. Issues of transgender men were not addressed due to their invisibility. Future studies should focus on issues and concerns of transgender men who are more stigmatized in Indian society. As reaching out to the transgender women and hijra population was a challenge, the study's sample size remained limited, restricting the generalizability of the findings. Therefore, the findings of this study should be interpreted in the context of the study. Prospective research can be more tailored and targeted to reach out to a maximum number of transgender individuals to mitigate this.

Acknowledgements The authors appreciate participants who expressed their deep emotions and greatly assisted the research work.

Author Contribution AP has conceptualized and coordinated the study. $\mathrm{AR}$ aided in the manuscript writing. Both authors have reviewed the manuscript and approved the final version.

Availability of Data and Material Data and material are available; however, participant data will not be shared due to confidentiality.

\section{Declarations}

Ethics Approval All procedures performed in studies involving human participants were in accordance with the ethical standards of the institutional and/or national research committee and with the 1964 Helsinki Declaration and its later amendments or comparable ethical standards. The study was approved by the research ethics committee of a community-based organization.

Consent to Participate All individual participants included in the study agreed after informed consent procedures. 
Consent to Publish The participants have consented to submit results that do not identify any participants to the journal for wider dissemination of the study findings.

Conflict of Interest The authors declare no competing interests.

\section{References}

Armitage, R., \& Nellums, L. B. (2020). COVID-19 and the consequences of isolating the elderly. The Lancet Public Health, 5(5), e256. https://doi.org/10.1016/S2468-2667(20)30061-X

Avafia, T., Konstantinov, B., Esom, K., Sanjuan, J. R., \& Schleifer, R. (2020, March 24). A rights-based response to COVID-19: Lessons learned from HIV and TB epidemics. Health and Human Rights Journal. https://www.hhrjournal.org/2020/03/a-rights-basedresponse-to-covid-19-lessons-learned-from-hiv-and-tb-epidemics/

Banerjee, D., Popoola, J., Shah, S., Ster, I. C., Quan, V., \& Phanish, M. (2020). COVID-19 infection in kidney transplant recipients. Kidney International, 97(6), 1076-1082. https://doi.org/10.1016/j. kint.2020.03.018

Braun, V., \& Clarke, V. (2006). Using thematic analysis in psychology. Qualitative Research in Psychology, 3, 77-101. https://doi.org/10. 1191/1478088706qp063oa

Cachia, M., \& Millward, L. (2011). The telephone medium and semistructured interviews: A complementary fit. Qualitative Research in Organizations and Management, 6(3), 265-277. https://doi.org/ $10.1108 / 17465641111188420$

Cortazzi, M. (2014). Narrative analysis. Routledge.

Centers for Disease Control and Prevention. (2020, April 15). Assessing risk factors for severe COVID-19 illness. https://www.cdc. gov/coronavirus/2019-ncov/covid-data/investigations-discovery/ assessing-risk-factors.html

Chakrapani, V. (2016). Sex change operation and feminising procedures for Transgender women in India. In N. Arvind, \& V. Chandra (Eds.), Nothing to fix: Medicalization of sexual orientation and gender identity (pp. 137-159). Sage.

Chakrapani, V., Vijin, P. P., Logie, C. H., Newman, P. A., Shunmugam, M., Sivasubramanian, M., \& Samuel, M. (2017). Understanding how sexual and gender minority stigmas influence depression among transwomen and men who have sex with men in India. LGBT Health, 4(3), 217-226. https://doi.org/10.1089/lgbt.2016. 0082

Chakrapani, V., Willie, T. C., Shunmugam, M., \& Kershaw, T. S. (2019). Syndemic classes, stigma, and sexual risk among transgender women in India. AIDS and Behavior, 23(6), 15181529. https://doi.org/10.1007/s10461-018-2373-1

Deb, S. (2020, March 26). Living on the edge: COVID-19 adds to distress and discrimination of Indian transgender communities. Health and Human Rights Journal. https://www.hhrjournal. org/2020/03/living-on-the-edge-covid-19-adds-to-distress-anddiscrimination-of-indian-transgender-communities/

Drabble, L., Trocki, K. F., Salcedo, B., Walker, P. C., \& Korcha, R. A. (2016). Conducting qualitative interviews by telephone: Lessons learned from a study of alcohol use among sexual minority and heterosexual women. Qualitative Social Work, 15(1), 118-133. https://doi.org/10.1177/1473325015585613

Economic Times. (2020, March 25). Gujarat Government to provide food items free to 60 lakhs poor families. Economic Times. https:// economictimes.indiatimes.com/news/politics-and-nat-nation/ gujarat-govt-to-provide-food-items-free-to-60-lakh-poor-families/ articleshow/

Editorial. (2020). Redefining vulnerability in the era of COVID-19. The Lancet, 395(10230), 1089. https://doi.org/10.1016/S01406736(20)30757-1
Elavati, M. (2016). Access to justice. People's Inquiry into the Status of the Implementation of the NALSA Judgment. Human Rights Law Network. http://reproductiverights.hrln.org/wp-content/uploads/ 2017/04/nalsa-ipt-report.pdf

Ganju, D., \& Saggurti, N. (2017). Stigma, violence and HIV vulnerability among transgender persons in sex work in Maharashtra, India. Culture, Health \& Sexuality, 19(8), 903-917. https://doi. org/10.1080/13691058.2016.1271141

Hall, K. S., Samari, G., Garbers, S., Casey, S. E., Diallo, D. D., Orcutt, M., \& McGovern, T. (2020). Centring sexual and reproductive health and justice in the global COVID-19 response. The Lancet, 395(10231), 1175-1177. https://doi.org/10.1016/S0140-6736(20) 30801-1

Hebbar, Y. R. N., \& Singh, B. (2017). Psychiatric morbidity in a selective sample of transgenders in Imphal, Manipur: A descriptive study. Annals of Indian Psychiatry, 1(2), 114-117.

Hegde, A. (2020, April 29). Keeping the lens focused: Gender and COVID-19. https://www.policycorps.org/post/keeping-the-lensfocused-gender-and-covid-19

Humsafar Trust. (2012). Feminisation and substance use in the maleto-female transgender/Hijra population in India: A needs assessment. https://archive.nyu.edu/handle/2451/33882

Irvine, A., Drew, P., \& Sainsbury, R. (2013). Am I not answering your questions properly? Clarification, adequacy and responsiveness in semi-structured telephone and face-to-face interviews. Qualitative Research, 13(1), 87-106. https://doi.org/10. 1177/2F1468794112439086

James, S. E., Herman, J. L., Rankin, S., Keisling, M., Mottet, L., \& Anafi, M. (2016). The Report of the 2015 U.S. Transgender Survey. National Center for Transgender Equality. https://www. transequality.org/sites/default/files/docs/USTS-Full-ReportFINAL.PDF

Jayadeva, V. (2017). Understanding the mental health of the Hijra women of India. American Journal of Psychiatry Residents' Journal, 12(5), 7-9. https://doi.org/10.1176/appi.ajp-rj.2017

Kim, H., Sefcik, J. S., \& Bradway, C. (2017). Characteristics of qualitative descriptive studies: A systematic review. Research in Nursing \& Health, 40(1), 23-42. https://doi.org/10.1002/nur.21768

Law, M., Mathai, A., Veinot, P., Webster, F., \& Mylopoulos, M. (2015). Exploring lesbian, gay, bisexual, and queer (LGBQ) people's experiences with disclosure of sexual identity to primary care physicians: A qualitative study. BMC Family Practice, 16, 175. https://doi.org/10.1186/s12875-015-0389-4

Lechuga, V. M. (2012). Exploring culture from a distance: The utility of telephone interviews in qualitative research. International Journal of Qualitative Studies in Education, 25(3), 251-268. https:// doi.org/10.1080/09518398.2010.529853

Ming, L. C., Hadi, M. A., \& Khan, T. M. (2016). Transgender health in India and Pakistan. The Lancet, 388(10060), 2601-2602. https:// doi.org/10.1016/S0140-6736(16)32120-1

Ministry of Health and Family Welfare. (2020). COVID-19 dashboard. https://www.mohfw.gov.in/

Miles, M. B., \& Huberman, A. M. (1994). Qualitative data analysis: An expanded sourcebook. Sage.

Muralidharan, S., Acharya, A., Koshy, A. V., Koshy, J. A., Yogesh, T. L., \& Khire, B. (2018). Dentition status and treatment needs and its correlation with oral health-related quality of life among men having sex with men and transgenders in Pune city: A cross-sectional study. Journal of Oral and Maxillofacial Pathology, 22(3), 443. https://doi.org/10.4103/jomfp.JOMFP_187_18

National AIDS Control Organization. (2017, April 15). HIV sentinel surveillance: technical brief, India 2016-17. Ministry of Health and Family Welfare, Government of India. http://naco.gov.in/sites/ default/files/HIV\%20SENTINEL\%20SURVEILLANCE_06_12_ 2017 0.pdf 
National AIDS Control Organization \& ICMR-National Institute of Medical Statistics (2020, April 15). India HIV Estimates 2019: Report. Ministry of Health and Family Welfare, Government of India http://naco.gov.in/sites/default/files/INDIA\%20HIV\% 20ESTIMATES.pdf

Ortiz, Z. (2016). The transgender community in Bangalore: Health issues and factors negatively affecting outcomes. https:// digitalcollections.sit.edu/isp_collection/2516/

Pandya, A., \& Redcay, A. (2021). Access to health services: Barriers faced by the transgender population in India. Journal of Gay \& Lesbian Mental Health, 25(2), 132-154. https://doi.org/10.1080/ 19359705.2020.1850592

Parveen, S. (2020, March 18). Coronavirus: LGBT community demands separate isolation wards. Asia News International. https://www. aninews.in/news/national/general-news/coronavirus-lgbtcommunity-demands-separate-isloation-wards20200318040746/

Roy, D., Tripathy, S., Kar, S. K., Sharma, N., Verma, S. K., \& Kaushal, V. (2020). Study of knowledge, attitude, anxiety and perceived mental healthcare need in Indian population during COVID-19 pandemic. Asian Journal of Psychiatry, 51, 102083. https://doi. org/10.1016/j.ajp.2020.102083

Saraswathi, A., \& Praveen, P. A. (2015). To analyze the problems of transgender in India: A study using new triangular combined Block Fuzzy Cognitive maps. International Journal of Science and Engineering Research, 6(3), 186-195.

Sharma, P. (2018, May 15). Only male or female can get PAN card, transgender. Times of India. http://timesofindia.indiatimes.com/ articleshow/63321785.cms?utm_source=contentofinterest\&utm_ medium $=$ text\&utm_campaign $=$ cppst
Singh, A. (2020, April 06). COVID-19 outbreak: Rural India stares at a larger crisis. Deccan Herald. https://www.deccanherald.com/ business/covid-19-outbreak-rural-india-stares-at-a-larger-crisis821866.html

Spoorthy, M. S., Pratapa, S. K., \& Mahant, S. (2020). Mental health problems faced by healthcare workers due to the COVID-19 pandemic- a review. Asian Journal of Psychiatry, 22, 102119. https:// doi.org/10.1016/j.ajp.2020.102119

Stop TB Partnership. (2018). A rapid assessment of gender and Tuberculosis in India. http://www.stoptb.org/assets/documents/ communities/CRG/TB\%20Data\%20Assesment\%20India.pdf

Thompson, L. H., Dutta, S., Bhattacharjee, P., Leung, S., Bhowmik, A., Prakash, R., Isac, S., \& Lorway, R. R. (2019). Violence and mental health among gender-diverse individuals enrolled in a human immunodeficiency virus program in Karnataka. South India. Transgender Health, 4(1), 316-325. https://doi.org/10.1089/trgh.2018.0051

Trier-Bieniek, A. (2012). Framing the telephone interview as a participant-centred tool for qualitative research: A methodological discussion. Qualitative Research, 12(6), 630-644. https://doi.org/ 10.1177/2F1468794112439005

World Health Organization. (2020). Coronavirus disease (COVID19) and advice for the public. WHO, Geneva. https://www.who.int/ emergencies/diseases/novel-coronavirus-2019/advice-for-public

Publisher's Note Springer Nature remains neutral with regard to jurisdictional claims in published maps and institutional affiliations. 